\title{
Cryptococcal meningitis in an HIV negative patient with systemic sarcoidosis
}

\author{
R J P Botha, E Wessels
}

\begin{abstract}
A case of Cryptococcus neoformans meningitis is described in an HIV negative patient with undiagnosed systemic sarcoidosis. The patient presented with signs of meningitis together with generalised lymphadenopathy and hepatosplenomegaly. Cryptococcal meningitis was diagnosed on lumbar puncture. She was treated with intravenous amphotericin $B$ but died within two weeks of admission. Necropsy revealed lesions in the lungs, liver, spleen, lymph nodes, small intestine, and bone marrow consistent with sarcoidosis. Microscopically the lesions contained non-caseating epithelioid cell granulomas typical of sarcoidosis. No Schaumann or Hamazaki-Wesenberg bodies were identified. Cryptococcus neoformans meningitis is generally associated with immunosuppressive disorders. As $\mathbf{T}$ cell abnormalities have been described in sarcoidosis, this could have been a case of opportunistic infection. Although rare, sarcoidosis merits consideration in patients with cryptococcal disease in the absence of HIV infection. (f Clin Pathol 1999;52:928-930)
\end{abstract}

Keywords: cryptococcal meningitis; sarcoidosis; immunodeficiency; HIV

\section{Case report}

A 49 year old black woman presented with complaints of fatigue and loss of weight. Clinical examination showed diffuse lymphaden13 July 1999

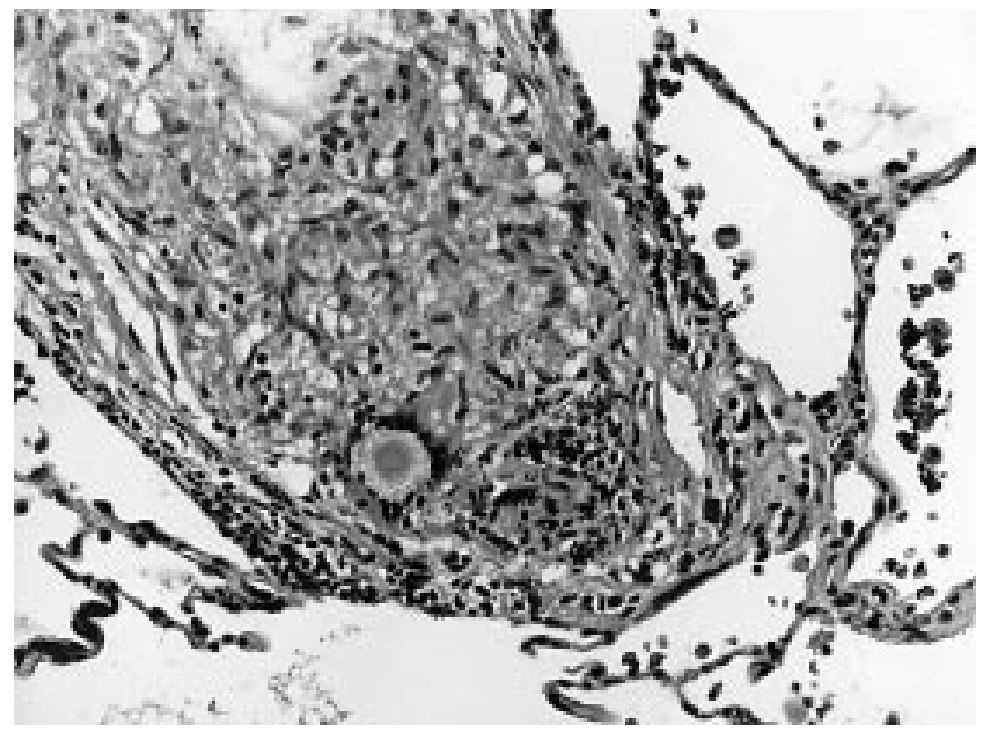

Figure 1 A high power view of a non-caseating epithelioid granuloma of the lung, showing a giant cell with an intracytoplasmic asteroid body. (Haematoxylin and eosin, $\times 563$.) opathy and hepatosplenomegaly. She seemed disorientated, and had terminal neck stiffness and fever. Her HIV status was negative. Her neurological status progressively deteriorated during the ensuing period of hospital admission. Computed tomography of the brain was normal. A lumbar puncture was performed and Cryptococcus neoformans was isolated in the cerebrospinal fluid. The patient was subsequently treated with intravenous amphotericin B.

A chest $x$ ray showed an interstitial infiltrate in the lingula of the left lung. Her serum angiotensin converting engyme level was raised. The patient did not respond to treatment and died two weeks after admission to hospital. A necropsy examination was requested.

The lungs, liver, spleen, lymph nodes, and bone marrow all showed comparable pathology at necropsy. Macroscopically the organs had widespread small firm white nodules on the surface as well as in the parenchyma. Microscopic examination (figs 1 and 2) showed numerous confluent non-caseating granulomas consisting of epithelioid cells and Langhans giant cells. Asteroid bodies were widespread in the cytoplasm of the giant cells. There were no signs of Schaumann or Hamazaki-Wesenberg bodies in any of the granulomas. These granulomas were located at the periphery of irregular areas of fibrosis. No acid or alcohol fast bacilli or fungal organisms could be demonstrated by Ziehl-Neelsen and Grocott's hexamine silver stains in any of the organs examined. The lymph nodes were enlarged and firm, with areas of calcification. The lymphoid tissue was diffusely replaced by dense fibrous connective tissue. The small intestine showed only a few small submucosal haemorrhages on macroscopic examination. Microscopically, moderate congestion and focal haemorrhages were seen, together with irregular areas of fibrosis containing scattered multinucleated giant cells in the submucosa. The appearance of these confluent granulomata was identical to those found in other organs. The rest of the intestinal wall was unaffected by the sarcoid disease process.

The subarachnoid space was filled with turbid cerebrospinal fluid. Microscopically a chronic inflammatory cell infiltrate consisting of lymphocytes, plasma cells, and histiocytes was present in the leptomeninges (fig 3). Abundant $C$ neoformans organisms were identified and clearly demonstrated by Grocott's stain. No intracerebral lesions were found. The cryptococcal infection was thus confined to the meninges as a diffuse non-granulomatous inflammatory process, in contrast to the sarcoid granulomata found in the viscera. No 


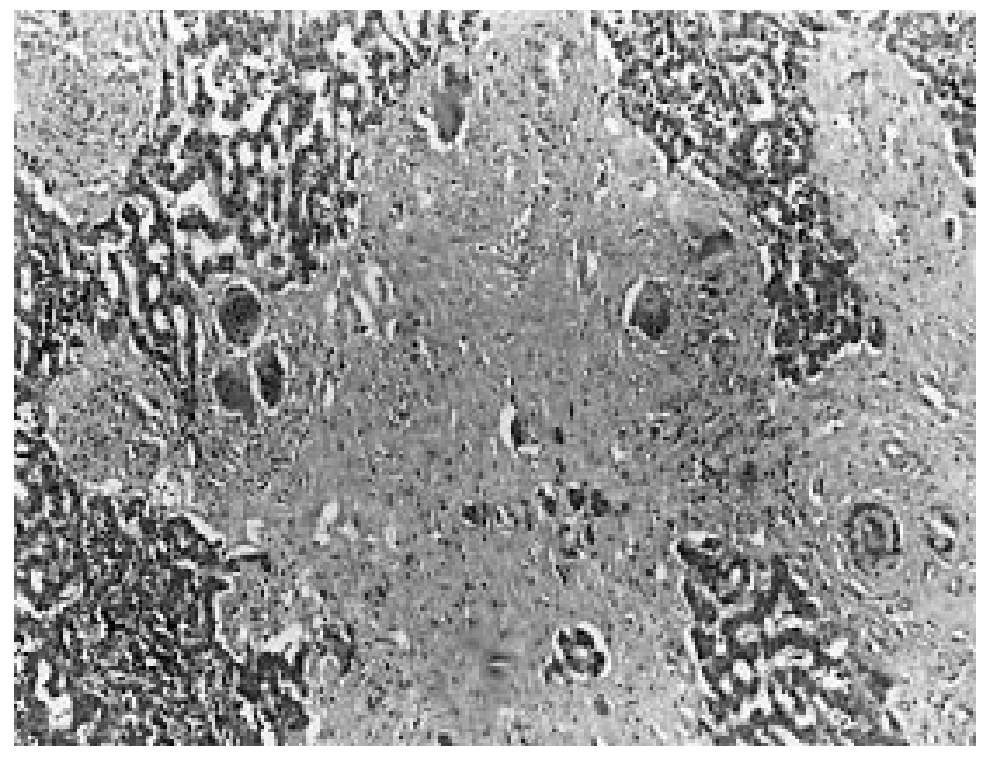

Figure 2 Liver with a large irregular area of fibrosis demonstrating numerous ill defined epithelioid granulomas with scattered giant cells. (Haematoxylin and eosin $\times 352$.)

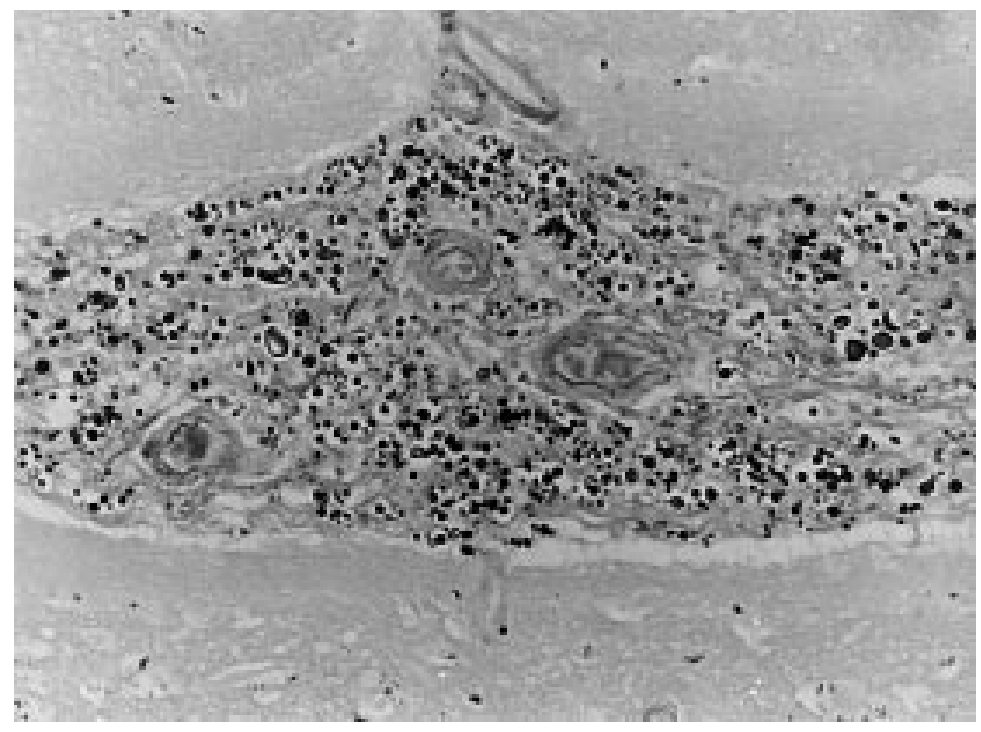

Figure 3 A high power view of the heavily colonised leptomeninges showing the characteristic morphology of Cryptococcus neoformans. (Grocott stain, $\times 563$.)

cryptococcal infestation was seen in any site outside the meninges.

\section{Discussion}

Sarcoidosis is a self limiting or chronic disease of unknown aetiology, although the current hypothesis is that it has more than one cause, and that each promotes a different pattern of illness. ${ }^{1}$ Various studies have suggested that sarcoidosis affects blacks more severely than people of other races. ${ }^{1}$ The prevalence of sarcoidosis in South African blacks is between 20.4 and 23.2 per 100000 of the population. This is higher than among the white population, and the figures are comparable with incidence rates in London. ${ }^{2}$

Belcher $e t a l{ }^{3}$ in a study of two cases of sarcoidosis complicated by cryptococcosis, state that up to the time of writing (June 1975) at least 25 cases of this disease combination had been reported. ${ }^{3}$ This indicates that it may be a worldwide and not excessively rare association.
Belcher's study, like many before and after, ${ }^{4-10}$ focused on the impaired cell mediated immunity of two patients with longstanding documented sarcoidosis who received steroid treatment (one topical and one systemic). Their course was complicated by the eventual development of cryptococcal osteomyelitis and pulmonary cryptococcosis. It was suggested that the administration of steroids had no direct causative role in the cryptococcal infection in patients with sarcoidosis, but that it might facilitate the dissemination of the infection. ${ }^{3-5}$ The impaired cell mediated immunity persisted long after cessation of steroid treatment. It was not possible to define the precise nature of the defect in cell mediated immunity but it was shown that the other host defence mechanisms (antibody production and neutrophil function) were normal.

The most common sites of cryptococcal infection in sarcoid patients are osteolytic bone lesions, pulmonary infection, and localised skin lesions, with a non-lethal course. Patients with $C$ neoformans in the cerebrospinal fluid have a high mortality. ${ }^{5}$ An unusual case of cryptococcal prostatitis was reported in 1972; this is rarely associated with sarcoidosis. ${ }^{6} \mathrm{~A}$ few cases have been published since the 1970 s with combinations of sarcoidosis and cryptococcosis together with other diseases such as cysticercosis, tuberculosis, and coccidioidomycosis. ${ }^{8} 1011$

Our case is one of a patient who probably contracted sarcoidosis many months or even years before presenting at our tertiary institution. Because of the unknown nature of her underlying disease she had never received any steroid or other treatment that we know of. She thus presented a diagnostic challenge to the physicians. Despite being seronegative for HIV, she appears to have been predisposed to opportunistic infection because of the sarcoidosis.

Patients with sarcoidosis have a decreased CD4/CD8 ratio, possibly because of the redistribution of helper $\mathrm{T}$ cells from the blood to the lungs and other sites of disease activity. ${ }^{12}{ }^{13}$ Sarcoidosis has also been described in association with common variable immunodeficiency disease (CVID), characterised by low serum immunoglobulin levels and impaired antibody responses. $\mathrm{T}$ cell abnormalities have also been described in CVID. ${ }^{13}$

Cryptococcosis, as an opportunistic $\mathrm{T}$ cell modulated mycosis, is an important disease in patients with a weak or suppressed immune system. ${ }^{7}$ It is a major cause of fungal meningitis and is often seen in patients with AIDS. ${ }^{14}$ However, cryptococcal meningitis can occur in immunocompetent hosts. Some investigators have found that $C$ neoformans, variety gattii, has a greater propensity to cause disease in immunocompetent hosts. ${ }^{15}$ Immunocompromised patients are almost exclusively infected by $C$ neoformans, variety neoformans. ${ }^{15}$

The specific variety of $C$ neoformans was not identified in our patient but was presumably variety neoformans in the light of her weakened immunity associated with sarcoidosis and the resultant susceptibility to opportunistic infection. However, single organ infection by 
cryptococcus in an immunocompromised host, as in this case, is unusual. Sarcoidosis merits consideration in HIV negative patients with cryptococcal infection.

1 Newman LS, Rose CS, Maier LA. Sarcoidosis. N Engl f Med 1997;336:1224-34.

2 Smith C, Feldman C, Reyneke J, et al. Sarcoidosis in Johannesburg - a comparative study of black and white patients. S A Med f 1991;80:423-7.

3 Belcher RW, Palazij R, Wolinsky E. Immunologic studies in patients with sarcoidosis and cryptococcosis. Arch Dermatol 1975;111:711-16.

4 Anonymous. A case of sarcoidosis with cryptococcal meningitis demonstrated at the Royal Postgraduate Medical School. BMF 1969;iv:729-32.

5 Nottebart HC, McGehee RF, Utz J. Cryptococcosis complicating sarcoidosis. Am Rev Respir Dis 1973;107: 1060-3.

6 Brock DJ, Grieco MH. Cryptococcal prostatitis in a patient with sarcoidosis: response to 5-fluorocytosine. F Urol 1972;

7 Bohne T, Sander A, Pfister-Wartha A, et al. Primary cutaneous cryptococcosis following trauma of the right forearm. Mycoses 1996;39:457-9.
8 Ahmad I, Sharma OP. Sarcoidosis, cysticercosis and cryptococcosis: an unusual association. Sarcoidosis 1989;6: 57-9.

9 Shijubo N, Fujishima T, Ooashi K, et al. Pulmonary cryptococcal infection in an untreated patient with sarcoidosis. Sarcoidosis 1995;12:71-4.

10 Shaff MI, Berger JL, Green NE. Cryptococcal osteomyelitis, pulmonary sarcoidosis and tuberculosis in a single patient. South Med f 1982;75:225-6.

11 Lord GP. Pulmonary sarcoidosis complicated by cryptococcosis and coccidioidomycosis. The changing spectrum of fungus disease in Maine. F Maine Med Assoc 1974;65:23640.

12 Fasano MB, Sullivan KE, Sarpong SB, et al. Sarcoidosis and common variable immunodeficiency. Medicine 1996;75: 251-61.

13 Hunninghake GW, Crystal RG. Pulmonary sarcoidosis: a disorder mediated by excess helper T-lymphocyte activity at sites of disease activity. $N$ Engl F Med 1981;305:429-34.

14 Dismukes WE. Cryptococcal meningitis in patients with AIDS. F Infect Dis 1988;157:624-8.

15 Mitchell DH, Sorrel TC, Allworth AM, et al. Cryptococcal disease of the CNS in immunocompetent hosts: influence of cryptococcal variety on clinical manifestations and outcome. Clin Infect Dis 1995;20:611-16. 